\title{
To the Question about the Rings on the Ice of Lake Baikal
}

\author{
Vasiliy Karlovich Balkhanov ${ }^{*}$ and Yuriy Buddich Bashkuev
}

\author{
Institute of Physical Materials Science of the Siberian Branch, of the Russian Academy of Sciences, \\ Ulan-Ude City, Russia
}

\begin{abstract}
More than 20 years in the monitoring of the Earth's surface with spacecraft in the spring on a snow-covered ice of Lake Baikal regularly observe giant rings with diameter of up to $8 \mathrm{~km}$. Most researchers agree that the appearance of rings connected with a different kind of activity of the lake bottom. This may be mud volcanoes, which are not uncommon in Baikal, or methane emissions of bottom sediments in the form of hot jets. Anyway, the determining factor for the appearance of rings are thermal processes. So as ice lies on the water, the thermal conductivity equations must also attract and equations of hydrodynamics. Thus, the study of rings on the lies lies solid basis of the mathematical description. The article gives some figures related to the rings. Briefly describes what led the joint consideration of the equations of fluid dynamics and heat transfer in the study of giant rings on a snow-covered Lake Baikal ice field.
\end{abstract}

Keywords: Lake baikal, Circular rings, The equations of fluid dynamics, Thermal physics.

\section{INTRODUCTION}

In March 2016, when analyzing imagery from Terra and Aqua satellites again found on the ice of Lake Baikal ring structure of Cape's Head (the southern tip of the peninsula of Svyatoy Nos) (Figure 1). Ring structure in ice cover of Lake Baikal does not appear every year. Thanks to constant monitoring of Lake professionals were able to identify the place of most frequent occurrence

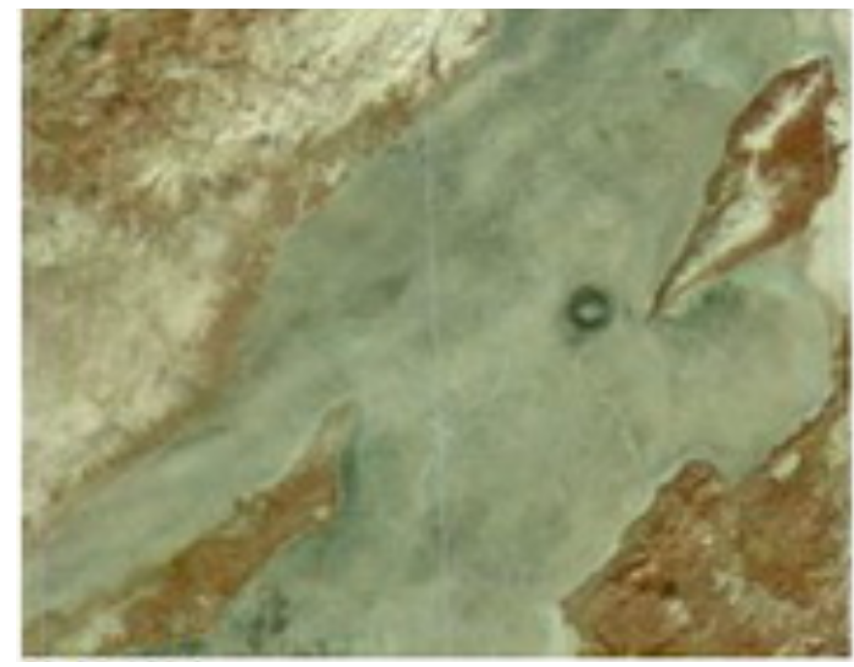

Figure 1: March, 2016.

of Baikal rings. They were first detected on satellite imagery at the end of the 90-ies of the last century. Then ring structure on the ice of Lake Baikal appeared in 2003 and 2005 respectively. In 2008, they appeared

"Address correspondence to this author at the nstitute of Physical Materials Science of the Siberian Branch, of the Russian Academy of Sciences, UlanUde City, Russia; Tel: 79834267532; Fax; 3012433224;

E-mail: ballar@yandex.ru in two parts of the Lake: in the area of the Krestovsky Cape and, for the first time in the region of the village of Turka. In 2009, they were discovered West of Cape's headboard and in the southern part of the Lake. In the year 2011 the pictures revealed three rings Krestovsky Cape, South of the island of Olkhon island and Cape's Headboard. In 2013, two rings at krestovsky Cape and Peninsula Svyatoy Nos. In the 2014 year year were detected only one ring, also from peninsula Svyatoy Nos [http://www.baikaldaily.ru/news/16/202234/] (Figure 2-3).

We are not demonstrate of all space images Baikal rings. This promise soon Irkutsk experts. History of the discovery of the rings on the ice of Lake Baikal is detailed in [1].

The emergence of giant rings on the ice of Lake Baikal calls active interest among the scientific community. So, G.S. Bordonskiy and co-authors [2] proposed the following mechanism of formation of the rings. Day and night temperature of ambient air is different. When you change the temperature of any material which is ice, shrinks and expands. This leads to deformation of the ice, and he begins to change their physical properties. Because the process happens on the plane. Because of the symmetry deformation takes the form of a circle. However, the proposed mechanism raises many questions. For example, why not occurs system concentric circles?

Previously, the presence of rings observed employees Limnological Institute of the RAS, in particular Nicholas G. Granin. He suggested that the rings can occur due to rise deep waters, which are called volcanic mud volcanoes. While in the central part of future ring structure rises temperature (an average of 


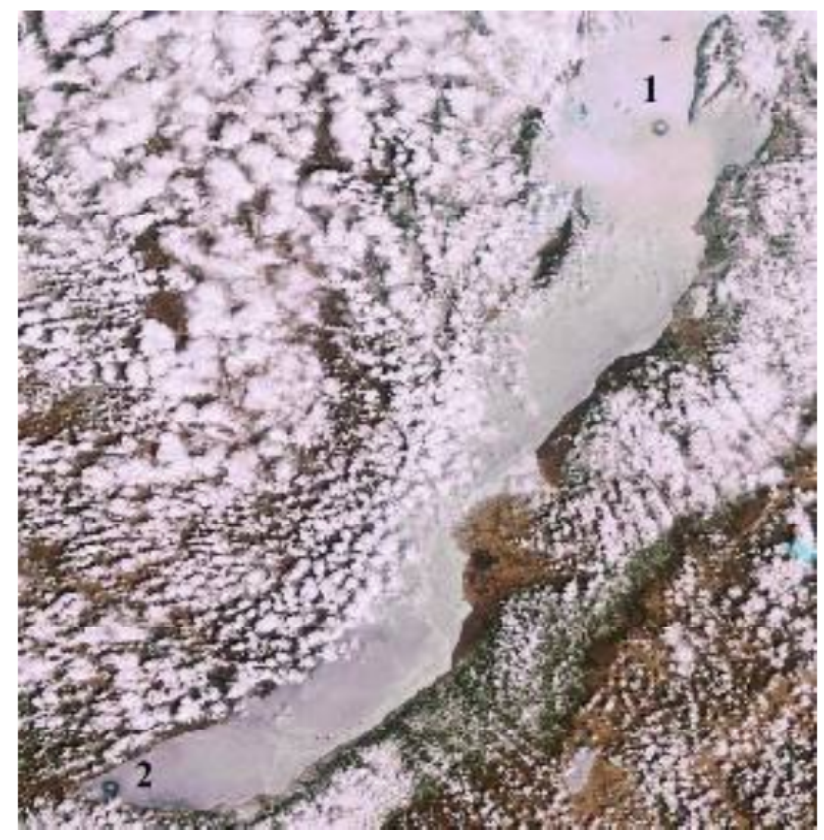

Figure 2: Circular ring 1 (near the peninsula of Svyatoy Nos) and 2 (near the southern tip of Lake Baikal) on Lake Baikal. Snapshot from 4.04.2009 [http://www.ocean.ru/content/view/784/75/].

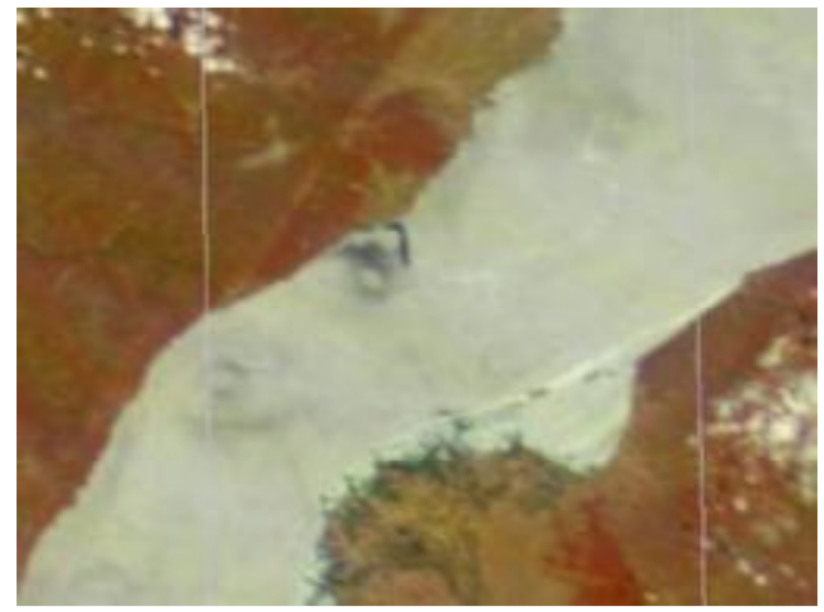

Figure 3: Rings in the area of krestovsky Cape.

Photo taken by the satellite "Terra" May 9, 2010 year.

half a degree, compared with other parts of the Lake), and formed the so-called anti cyclone (for a against circular movement of the time arrows). For increases vertical water exchange. As a result, the ice cover collapses stronger [3].

The authors agree that the main factor of education rings are heat flows from the bottom of the Lake. We follow a simple hypothesis - convection column of water in the form of a giant Thor due to uplift a jet of mineral gas (Figure 4-5).

In the works [4.5] installed equation, describing the convection of the water column in the form of giant
Tora. Equations are derived using the equations of fluid dynamics (Navier-Stokes equations), and the heat equation. Thus, the formation of ice on the surface of the rings we associate with a giant convection throughout the thickness of water around the emission jets of warm gas. In the middle rings of the ice water temperature above, on $0.5{ }^{\circ} \mathrm{C}$, from the rest of the water, observed N. G. Granin. This leads to melting during the month of snow and ice. Water of Lake Baikal is characterized by the fact that in the spring the temperature throughout the depth of practically does not change and is usually $T_{0}=3.2-3.4{ }^{\circ} \mathrm{C}$. Only near the surface, on depth of $150-200 \mathrm{~m}$ temperature 


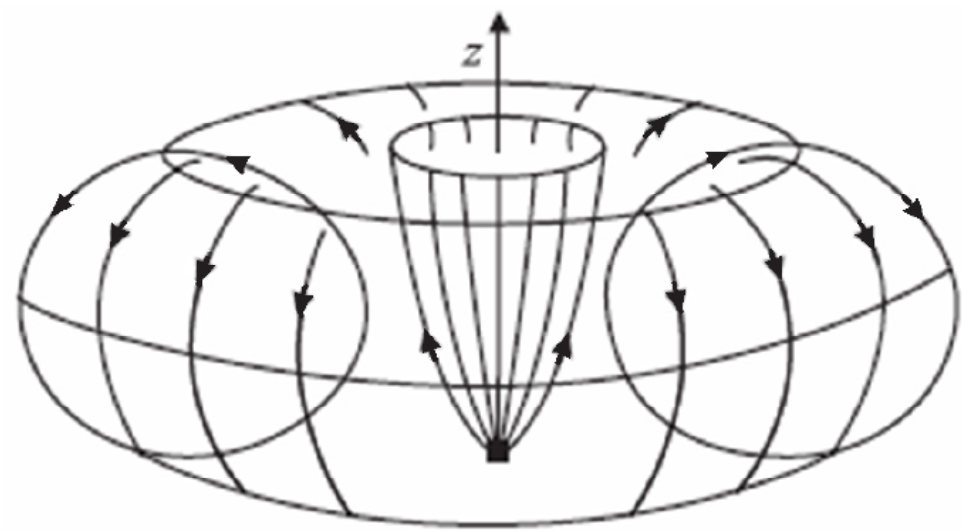

Figure 4: Lift warm jets of natural gas and convective water flow around jet in the form of a squashed Tora.

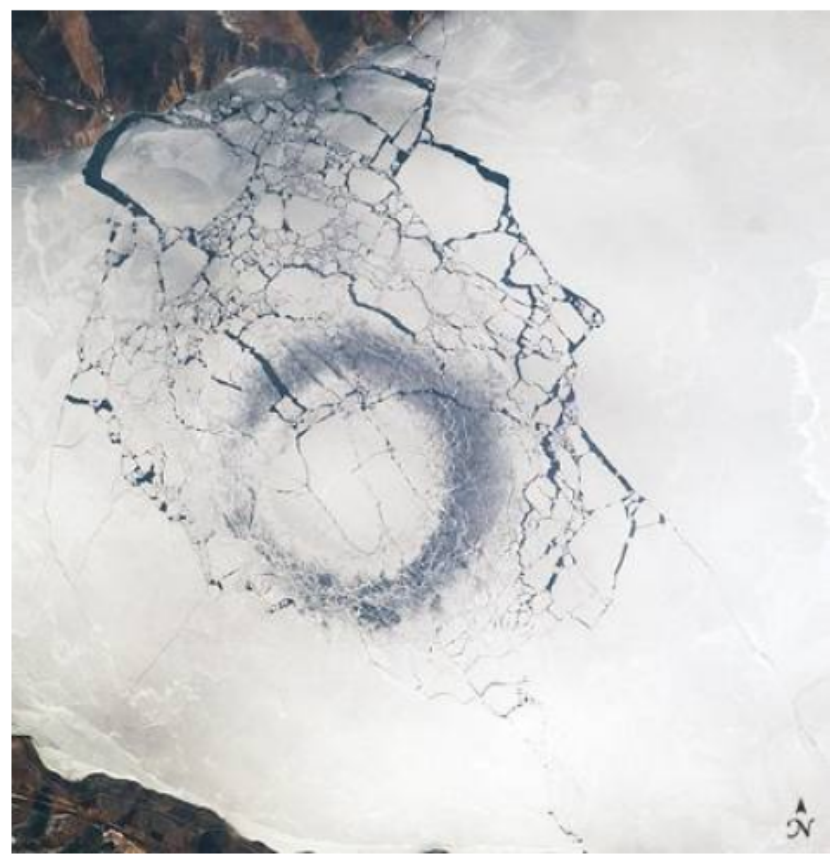

Figure 5: The destruction of the ice fields in result warmup ice near the southern tip of Lake Baikal. Photography from the International Space station "MKC" from 25.04.2009 [http://miravi.eo.esa.int/en/].

smoothly, albeit with minor variations, is reduced almost to $0{ }^{\circ} \mathrm{C}$ on the surface (see clauses Sherstyankin and others [6, 7]).

The resulting temperature gradient is so negligible that vertical convection of water does not occur. (We distract ourselves from internal currents in the water column, not related to thermal convection). Already you can see that the task is significantly different from the well-known Rayleigh-Benard convection. However, the expiration of the jet of warm natural gas of sedimentary strata bottom violates the mechanical equilibrium of water. Thickness of water comes in convective flow, which in effect took the form of symmetry, which is close to the Tora, as shown in Figure 5. Contact at the lower point with warmer natural gas, as a result of vertical rotation column water heat reaches the top point. This heat and spent melting ice, which eventually becomes thin and is saturated with water (Figure 6). As a result of space we see a dark ring.

Law rise the hot Jet still in the 30 -ies were clarified by the Academician Yakov Zeldovich [8]. A perfect analogy presented in Figure 6 and Figure 7 the explosion of a hydrogen bomb in the atmosphere and convection of the water column in the form of the Torah, as shown in Figure 4. This is not surprising, since both phenomena are described by the same equations of hydrodynamics and thermal conductivity. 


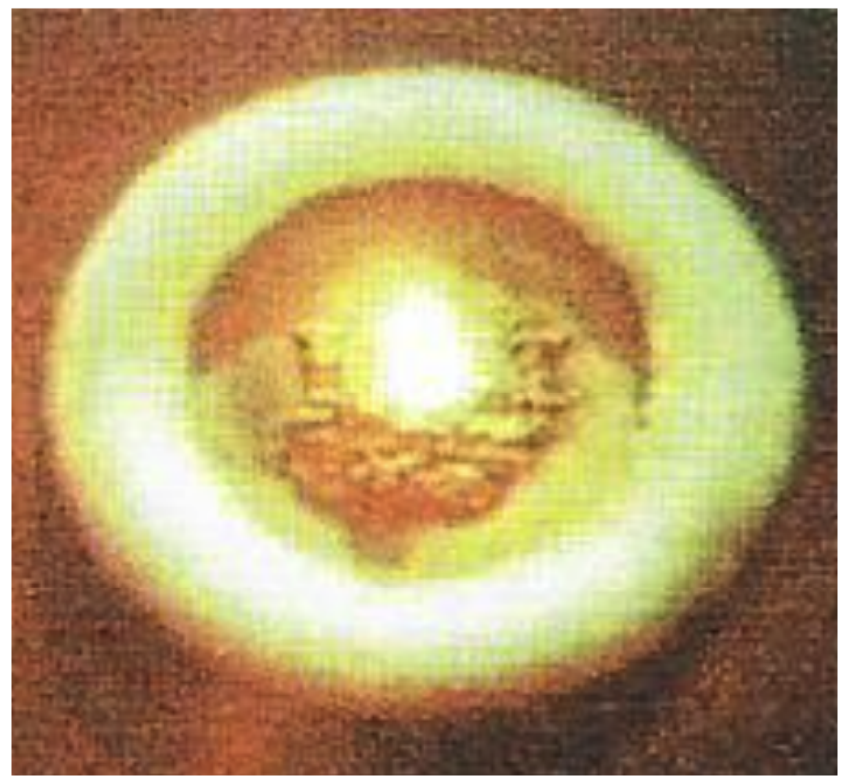

Figure 6: Explosion of a hydrogen bomb. View from the top. http://izvestia.ru/hystory/article3142873/.

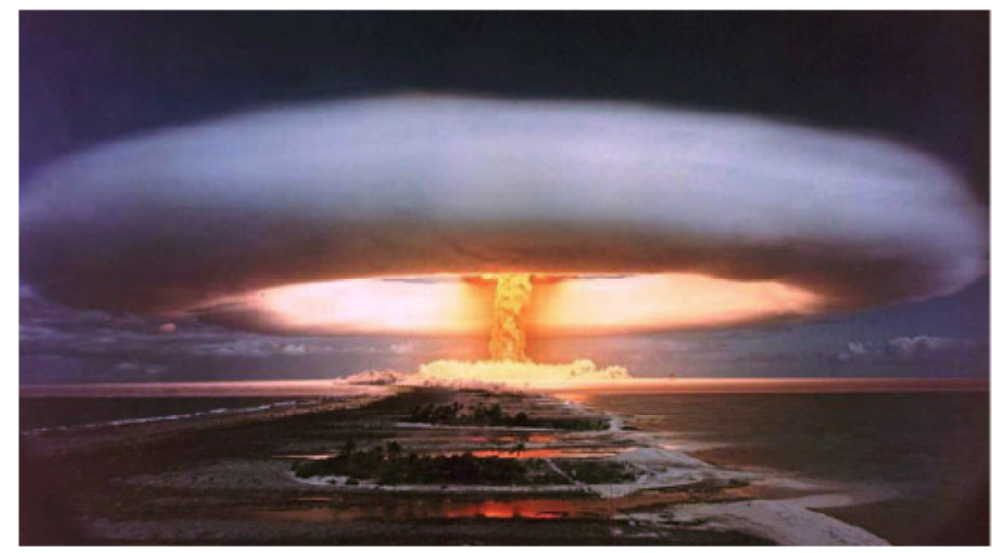

Figure 7: Explosion of a hydrogen bomb. Side view.

Therefore, the study of rings at Lake Baikal goes from the accumulation of observation of the facts in the area of the strictly mathematical description. Although there are still many unexplored. Lake Baikal geological plot rift zone of the Earth's crust (Rift is a large linear tectonic structure of the Earth's crust, it is the crack length hundreds of thousands of miles). Geological and geophysical data found that the Baikal rift zone refers to inland areas stretching of the Earth's crust and is located on the articulation of two contrasting thermal and mechanical properties of lithospheric: the Siberian platform and Central-Asian mobile belt. Basin are characterized by elevated heat flow and seismic activity, and Rift basin - powerful thickness of sedimentary rocks, where the accumulated plenty of organic matter. Increased thermal field and temperature gradient stipulate an intensive gas formations. Natural gas outputs, in particular methane, from the bottom of the Lake in the summer there are due to rising water surface bubbles. In winter time the formation of "proparin" (ice - free surface water) of size 0.5-100 $\mathrm{m}$ across, especially dangerous for cars. In addition to such relatively small "proparin", satellite imagery of Lake Baikal detected abnormally dark rings large size-diameter of 7-8 km, which mentioned above.

That education plays a decisive role rings it is heat flux can be seen in Figure 6, which shows how the temperature on the broken strength of the ice, and he destroyed on ice blocks of various sizes.

\section{EQUATION OF CONVECTIVE CURRENTS}

Let's consider the reasoning leading to a system of equations that describe of convection. Believe that the convection is a stationary process, in which all values do not depend explicitly on time. For such variables 
private time derivative becomes zero. In the absence of an external heat source water hasa mass of stationary temperature distribution $T_{0}$, depends only on location in depth (near the lake bottom $T_{0}=3.4^{\circ} \mathrm{C}$, on the surface of the water $T_{0}=0^{0} \mathrm{C}$ ). Convection occurs because in the liquid is introduced extraneous heat source. As a result, the stationary distribution of temperature $T_{0}$ added a small of temperature $T\left(\approx 0.5^{0} \mathrm{C}\right)$.

A convective motion occurs with slow speed. So speed $V$ convective currents is the value of a first order infinitesimal as a small additive of temperature $T$.

In the absence of an external heat source water density is constant and equilibrium value $\rho_{0}$. Under the ice interval of temperatures on Lake Baikal $\left(0-3.4{ }^{\circ} \mathrm{C}\right)$, when heated the water density is increasing. The first order of approximation by small additive of temperature $T$, resulting from the emergence of an external heat source, additive and density will be proportional to the temperature $T$. Thus, density $\rho$ becomes equal to $\rho=\rho_{0}+\beta \rho_{0} T$. Here the multiplier $\beta$ is called the coefficient of thermal expansion. In the interval from $0{ }^{0} \mathrm{C}$ before $3.4{ }^{\circ} \mathrm{C}$ it can be considered permanent.

In the absence of an external heat source pressure in water there is $P_{A}+\rho_{0} g(H-z)$, where is $H$ - Lake depth coordinate $z$ counting up from the bottom, $g-$ acceleration of free fall, which even for Baikal depths can be considered constant and equal to the Earth's surface, $P_{A}$ - the atmospheric pressure on the surface of the water. The emergence of a source of heat in the water makes the additional pressure $P$, so that the total pressure becomes $P_{A}+\rho_{0} g(H-z)+P$.

Taking into account all provisions in the first order $P$, $T$ and $V$ from the equations of fluid dynamics and heat transfer we get the following equations set describing convection:

$$
\begin{aligned}
& \nabla^{2} T_{0}=0 . \\
& (\vec{V} \cdot \nabla) T_{0}=\chi \nabla^{2} T . \\
& \nabla \cdot \vec{V}=0 . \\
& v\left(\nabla^{2}\right)^{2} \vec{V}=\beta(\vec{g} \cdot \nabla) \nabla T-\beta \vec{g} \nabla^{2} T .
\end{aligned}
$$

In here $v$ - viscosity of water, $\chi$ - thermal diffusivity. These both factors consider permanent. Verbose output of these equations is given in $[4,5]$, it is the same conclusion equations that describe RayleighBenard convection [8].

Mathematical analysis of the received results in the following system of equations $[4,5]$.

Near the water surface, the spatial distribution of temperature has a maximum value on the target distance from axis rise natural gas. This explains the melting ice in the form of rings. It has also been installed and the direction of rotation of the water column in Thor shape, as shown by the arrows in the Figure 5.

We have also considered the question of the criteria for occurrence volatility column of water in the form of a Thor vortex around the release of natural gas from the bottom of Lake Baikal. For Rayleigh-Benard flow a similar question is set out in [8]. Instability occurs when the perturbation solution for speed and temperatures begin to grow exponentially. Therefore, solving equations (1) to (4) were in exponential form. While for some the five functions are obtained only 4 equations. Thus these equations can be satisfied only after the advent of critical values for the five variables. The emergence of these critical values and means the emergence of convection in the form of a toroidal vortex in the water of Lake Baikal. In particular, the dimensionless critical Rayleigh number (known in fluid dynamics) turns out to be equal to approximately 1700 . Us also found that the size of the rings is directly proportional to the depth of the Lake (by a factor of 2.3), on which appear the Baikal rings. The latter means that if depth is equal to $1 \mathrm{~km}$, radius of the rings in this place is $2.3 \mathrm{~km}$. Since the maximum depth of the Lake is $1.7 \mathrm{~km}$, the maximum diameter of the ring will be $7.8 \mathrm{~km}$. In fact, the bottom of Lake Baikal is characterized by roughness, so the number of rate of 2.3 will weakly vary from place to place.

Scarce experimental data (actually only used satellite imagery and bathymetric map) do not permit until a more detailed analysis of the system of equations describing the phenomenon of formation of giant rings on snow - covered ice of Lake Baikal. More information would be useful on the physical characteristics of the natural gas jet, which is not yet available.

\section{CONCLUSION}

Over 20 years of monitoring of the Earth's surface with spacecraft have accumulated substantial material on the giant rings on a snow-covered field in Lake Baikal. Can be considered established that education is 
associated with thermal processes in the water of Baikal. Various researchers disagree about such sources of thermal processes. Either this is emissions of mud volcanoes, or this jet methane from bottom sediments. It is essential that the description given in the text of the convection equations does not depend on the nature of the external source of supplementary heat source. The only important thing is the presence. Although to date these equations is not yet fully explored, but available solutions indicate that they satisfactorily describe some features of giant rings. We believe that as long as sufficient and proposed equations. In any case, the study and description of considered rings received a solid mathematical basis.

\section{ACKNOWLEDGMENTS}

Article prepared for the budget draft Laboratory Electromagnetic Diagnostics Institute of Physical Materials Science of the Siberian Branch RAS.

\section{REFERENCES}

[1] Kouraev AV, Zakharova EA, Rémy F, Kostianoy AG, Shimaraev MN, Hall NMJ, Suknev A Ya. Giant ice rings on
Lakes Baikal and Hovsgol: inventory, associated water structure and potential formation mechanism, Limnology and Oceanography 2016; 61: 1001-1014. https://doi.org/10.1002/Ino.10268

[2] [in Russia] Bordonskiy GS, Krylov SD. On the nature of the ring formations on satellite images of the ice cover of Lake Baikal, The study of Earth from space 2014; pp. 27-31.

[3] [in Russia] Granin NG, V'sht A, Yu R, Gnatovskiy et al., IV Vereshaginskiy Baikal Conference 26 September - 1 October, year 2005, pp. 52-53.

[4] Balkhanov VK, Bashkuev Yu, Khaptanov VB. Formation of Circular Rings on the Snow-Covered Ice Field of Lake Baikal, ISSN 1063-7842, Technical Physics 2010; 55(9): 1266-1269. https://doi.org/10.1134/S1063784210090057

[5] Balkhanov VK, Bashkuev Yu. On the Appearance of a Convective Toroidal Vortex in Lake Baikal, Technical Physics 2013; 58(11): 1582-1586. https://doi.org/10.1134/S1063784213110054

[6] [in Russia] Sherstyankin PP, Fialkov VA, Chernyaev ES et al., Thermodynamic properties of convective and Baikal waters in the area of maximum depths of Lake Baikal observed with manned spacecraft "WORLD-2" during the spring termobara, Reports of the Academy of Sciences 2012; 446(6): 680-685.

[7] [in Russia] Drizhenko FK. Pilot and physico-geographical essay on Lake Baikal, Report of the Chief of the Hydrographic expedition of the Baikal Lake, (1908).

[8] Landau LD, Lifshitz EM. Fluid Mechanics, Oxford: Pergamon Press 1987.

Received on 20-11-2017

Accepted on 24-05-2018

Published on 12-06-2018

http://dx.doi.org/10.15379/2408-977X.2018.05.01.01

(C) 2018 Balkhanov and Bashkuev; Licensee Cosmos Scholars Publishing House.

This is an open access article licensed under the terms of the Creative Commons Attribution Non-Commercial License (http://creativecommons.org/licenses/by-nc/3.0/), which permits unrestricted, non-commercial use, distribution and reproduction in any medium, provided the work is properly cited. 